\title{
Quantum optical state comparison amplification of coherent states
}

Ugo Zanforlin, Ross J. Donaldson, Luca Mazzarella, Robert J. Collins, John Jeffers, et al.

Ugo Zanforlin, Ross J. Donaldson, Luca Mazzarella, Robert J. Collins, John Jeffers, Gerald S. Buller, "Quantum optical state comparison amplification of coherent states ," Proc. SPIE 10674, Quantum Technologies 2018, 1067413 (29 May 2018); doi: 10.1117/12.2307820

SPIE. Event: SPIE Photonics Europe, 2018, Strasbourg, France 


\title{
Quantum optical state comparison amplification of coherent states
}

\author{
Ugo Zanforlin*a, Ross J. Donaldson ${ }^{\mathrm{a}}$, Luca Mazzarella ${ }^{\mathrm{b}}$, Robert J. Collins ${ }^{\mathrm{a}}$, John Jeffers ${ }^{\mathrm{b}}$, \\ Gerald S. Buller ${ }^{\mathrm{a}}$ \\ a'SUPA, Institute of Photonics and Quantum Sciences, David Brewster Building,

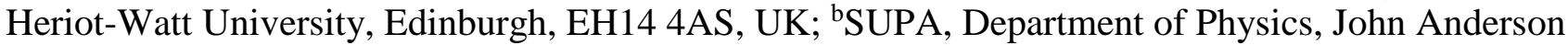 \\ Building, University of Strathclyde, 107 Rottenrow, Glasgow, G4 0NG, UK
}

\begin{abstract}
As light propagates through a transmission media, such as an optical fiber, it experiences a length-dependent loss which can reduce the communication efficiency as the transmission distance increases. In conventional telecommunications, optical signals can be transmitted over inter-continental distances, due to deterministic all-optical amplifiers. However, quantum communications are still limited to transmission distances of typically a few 100's km since deterministic amplifiers cannot be used to amplify quantum signals. The use of deterministic amplification on a quantum signal will introduce noise that will mask the original quantum properties of the signal, introducing uncertainty or errors to any measurement. Nondeterministic methods for amplifying quantum signals via post-selection can be used instead, providing a solution to create a low noise quantum amplifier. Several methods for nondeterministic amplification have already been experimentally demonstrated. However, these devices rely on "quantum resources" which makes implementation challenging. Here we present an overview of experimental demonstrations for amplifying coherent states using a method called state comparison amplification. This is a nondeterministic protocol that performs amplification of known sets of phase-encoded coherent states using two modular stages. The outcome of each stage is recorded using single-photon detectors and time-stamped electronics to enable post-selection. State comparison amplification is a relatively simple technique, only requiring "off-the-shelf" components. The presentation will show several demonstrations of state comparison amplification including an amplifier which has high gain, fidelity, and success rate with the added advantage of being robust to channel noise and easily reconfigurable. Finally, we will discuss the effect of introducing a feedforward mechanism allowing for unsuccessful state amplifications.
\end{abstract}

Keywords: quantum amplifier, state amplification, coherent states, state comparison, probabilistic amplifier, quantum technologies, optical amplifier

\section{INTRODUCTION}

\subsection{Quantum amplification}

The cryptographic security of many quantum communication protocols, such as quantum key distribution $(\mathrm{QKD})^{[1],[2],[3],[4]}$, quantum digital signature (QDS) ${ }^{[5],[6],[7],[8],[9]}$ and quantum bit commitment ${ }^{[10]}$, partially relies on the use of low-intensity optical coherent states, i.e. highly attenuated laser pulses. Optical signals propagating through transmission media, such as an optical fiber, are affected by a loss which is dependent on the overall length of the communication channel. Commercially available all-optical amplifiers can counteract the loss by exploiting deterministic optical effects ${ }^{[1]}$ allowing inter-continental transmissions. However, such amplifiers introduce noise levels ${ }^{[12]}$ which destroy any quantum properties that the coherent states carry making it challenging to perform quantum communication protocols over long distances. Over the last two decades, new and revised quantum cryptographic protocols have tried to address the problem ${ }^{[13]}$, although communication channel attenuation still poses a major limiting factor reducing the overall transmission distance of quantum communications to hundreds of kilometers ${ }^{[3],[14]}$.

*uz2@hw.ac.uk; phone +44 (0)131 451 4687; www.single-photon.com

Quantum Technologies 2018, edited by Jürgen Stuhler, Andrew J. Shields,

Miles J. Padgett, Proc. of SPIE Vol. 10674, 1067413 • ( 2018 SPIE

CCC code: $0277-786 X / 18 / \$ 18 \cdot$ doi: $10.1117 / 12.2307820$ 


\subsection{Nondeterministic quantum amplifier}

Although deterministic amplification of an unknown quantum state is prohibited by quantum mechanics ${ }^{[15],[16]}$, it is possible to address the problem with a probabilistic approach. Probabilistic amplification, also known as nondeterministic amplification, while not breaking any intrinsic quantum limit, is capable of amplifying quantum states by conditioning the amplification on specific detection events. Many different probabilistic amplifiers have been devised and experimentally demonstrated such as photon addition and subtraction ${ }^{[17]}$, random-noise addition combined with photon subtraction ${ }^{[18],[19]}$, stimulated parametric down conversion ${ }^{[20]}$, heralded scissor devices ${ }^{[21]}$, entanglement-based devices $^{[22]}$ or doped fibers ${ }^{[23]}$. These systems generally show low success probability, low generation rate and limited fidelity, i.e. a measure of the difference between the amplified state and input state, while requiring quantum components that are challenging to implement or require high precision control (such as photon number resolving detectors), inefficient post-selection criteria, and heralded single-photon sources. They also show low success probability and limited operating rates which makes them unsuitable for many quantum communication protocols ${ }^{[24]}$. On the contrary, state comparison amplifiers are capable of producing amplified quantum states with high-fidelity and high success rate because they efficiently condition the detection events on the available partial knowledge about the system ${ }^{[25]}$.

\subsection{Overview}

In this paper, we present different implementations of a probabilistic amplifier which strongly aligns with photon addition and subtraction devices both in structure and application but only relies on easily deployable "off-the-shelf" commercial components. The amplifier also shows high gain, improved fidelity and a high success rate which outperforms similar probabilistic amplifiers by several orders of magnitude. The first section will cover the basic principles of state comparison amplification and how it relates to this amplifier. The second section will present an overview of the experimental results focusing on three different configurations, i.e. low gain, high gain and amplification with added noise. The third section will introduce possible future works followed by a brief conclusion.

\section{STATE COMPARISON AMPLIFICATION}

State comparison amplification operates using the combined underlying principles of photon addition and subtraction ${ }^{[17]}$. As the combination suggests, state comparison amplifier performs quantum state amplification via a two-stage process. In the first stage, photons from an external source are added to the quantum state increasing the overall amplitude of the signal, hence the name photon addition. Following the photon addition stage is the subtraction stage where a vacuum state is interfered with the amplified signal, providing both an improvement to the amplifier's fidelity and suggestion of a successful amplification event. This technique usually adopts a low-reflectivity beam-splitter (BS) and a conditioning single-photon detector. Comparison amplifiers utilize a discrete, and known, set of quantum states over which they perform imperfect quantum nondemolition (QND) measurements ${ }^{[26]}$.

The state comparison amplifier (SCAMP) reported in this paper used a set of phase-encoded coherent states and comprised two standard stages: a state comparison stage and a state subtraction stage. The comparison stage performed an interferometric measurement between two coherent states on a BS. One of the states was a low-intensity coherent state with a randomly chosen unknown phase encoding which mimicked a quantum signal sent from a distant user, i.e. the input state, the other was a guess state generated by the amplifying node, i.e. the amplifier unit. The users had knowledge of the phase-encoding alphabet used by the device but did not share any information about the phase encoding used for each single quantum state between themselves. The resulting output of the measurement was monitored by a single-photon detector (SPD) operated in Geiger-mode whose detection events became post-selection criteria for subsequent analysis conditioning. When the input state and the guess state shared the same quantum properties, i.e. phase-encodings and amplitude, the system underwent destructive interference, no photons were detected by the SPD and the amplified signal was sent to the state subtraction stage.

Conditioning the system only on the SPD's output would provide a low-fidelity signal as component losses, non-unity detection efficiency, and a non-zero probability of a multiphoton pulse would suppress valid detection events especially at low intensities. For this reason, the state subtraction stage provided a secondary post-selection condition that increased the output fidelity at the cost of a reduced successful probability and lower gain. The stage comprised a hightransmissive (low-reflective) BS and an SPD operated in Geiger-mode. Here the condition for a successful amplification is met when a signal is detected by the SPD as a correctly amplified state is more likely to trigger a detection event than a wrongly chosen guess state. The SCAMP defines a successful amplification as the case when the SPD in the state 
comparison stage does not register an event and the SPD in the state subtraction stage does register an event, thereby confirming that it is likely the guess state and the input state share the same quantum properties.

The success of the SCAMP as a reliable and high-performance probabilistic amplifier is defined by two key parameters: correct state fraction (CSF) and interferometric visibility. CSF defines the proportion of states that have been correctly amplified against the total number of successfully correlated events. The interferometric visibility defines the ability of the system to discriminate between a correctly chosen guess state from a wrongly chosen one.

\section{METHOD AND RESULTS}

This section is divided into three subsections, each of which reports the experimental results of one of three different SCAMP configurations which vary both in post-selection criteria and optical setup. The first subsection presents the initial SCAMP setup and experimental results ${ }^{[27]}$ highlighting the achieved correct state fraction, i.e. correctly amplified quantum states, from state comparison techniques. The second subsection shows a SCAMP setup with an added state subtraction stage which allows higher overall gain at the cost of lower successful probability. The third, and final, subsection focuses on the amplifier performances when external broadband noise is added to the input quantum state.

\subsection{SCAMP}

Fig. 1 shows the experimental setup. A vertical-cavity surface-emitting laser (VCSEL) operated at a central wavelength of $849.8 \mathrm{~nm}$, generating weak coherent states (after attenuation by a motorized optical attenuator) at a clock frequency of $1 \mathrm{MHz}$. The optical pulses are then focused into a $5 \mu \mathrm{m}$ core diameter single mode polarization maintaining fiber. An operating wavelength around $850 \mathrm{~nm}$ was chosen because of a combination of the attenuation profile of optical fiber ${ }^{[28]}$ and the availability of easily deployable, highly efficient, low dark count, room temperature silicon single photon avalanche diodes (Si-SPADs) ${ }^{[29],[30],[31]}$. The optical setup was made of two interwoven interferometers: the innermost performed the state comparison amplification procedure while the outer one conducted a state tomography reconstruction between the input state and the amplified output. The guess state was phase modulated by a lithium-niobate electro-optic phase modulator which selected the phase randomly from a known discrete alphabet of $N$ phase encodings described by $2 \pi k / N$, where $k=0, \ldots, N-1$. In this realization, three different sets of phase encodings were used, $N=2,4$ and 8 .

A 50:50 BS (BS1) performed a QND measurement on the input and guess states while a Si-SPAD in Geiger-mode $\left(\mathrm{D}_{0}\right)$ monitored the output result. These two components comprised the amplifier's state comparison stage. When both input and guess states share the same phase and amplitude they interfere destructively, no signal is detected by $\mathrm{D}_{0}$, and the amplified output is redirected to the state subtraction stage which comprises a 90:10 BS (BS2) whose low-reflective port was monitored by a second Si-SPAD in Geiger-mode $\left(\mathrm{D}_{1}\right)$. The highly transmissive port of BS2 propagated the amplified quantum states to the tomography stage where an amplified copy of the original input state is interfered with the SCAMP-amplified state and the operating parameters of the SCAMP evaluated by considering the electrical outputs

of detectors $\mathrm{D}_{\mathrm{A}}$ and $\mathrm{D}_{\mathrm{B}}$. Fine-tuning of the relative optical paths of both interferometers is possible by using manually controlled adjustable air-gaps. 


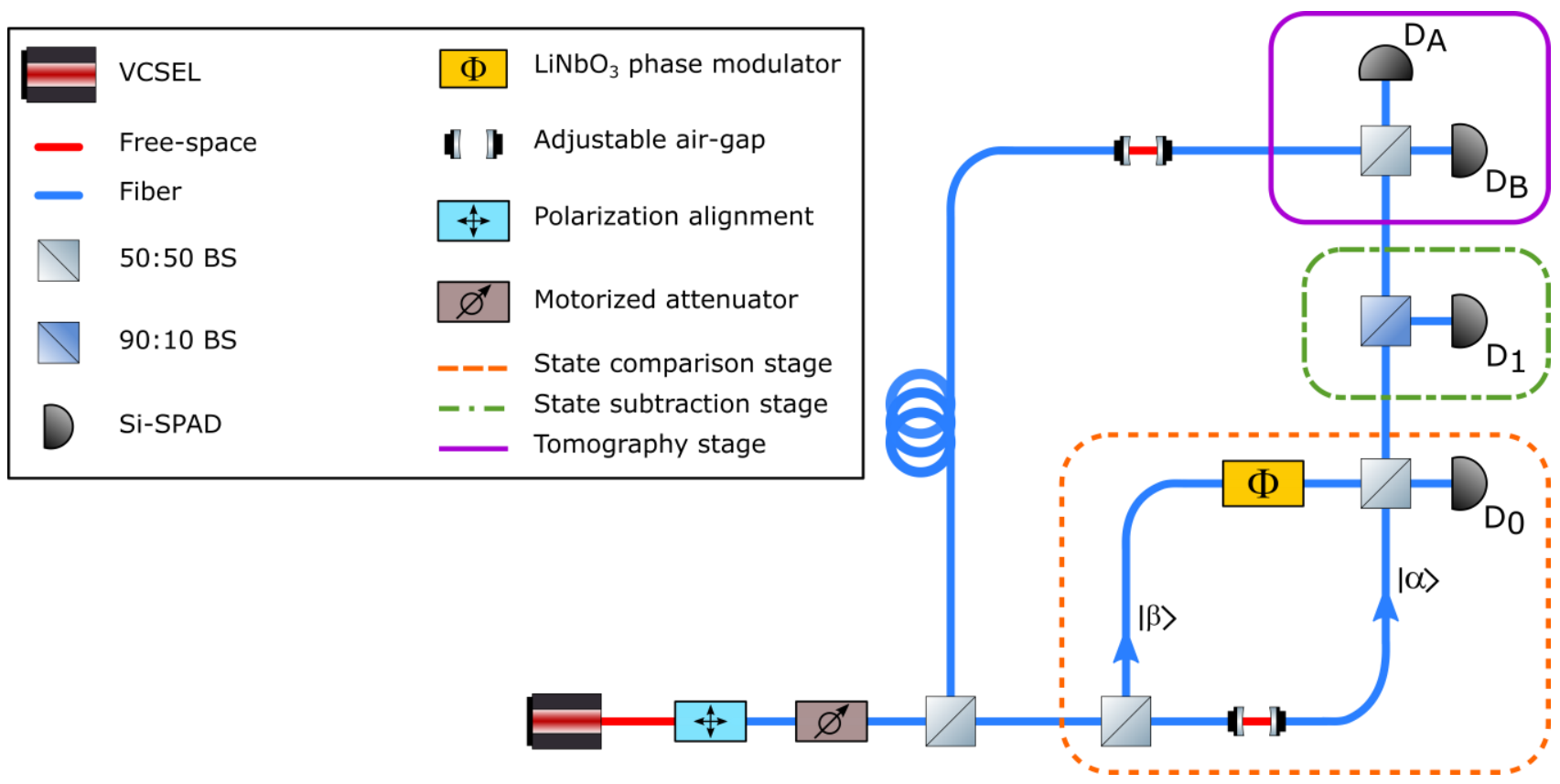

Figure 1. Experimental optical setup of the state comparison amplifier (SCAMP). The state comparison stage (enclosed within a dashed orange line) performs a nondemolition measurement of the phase of the input state $(|\alpha\rangle)$ and guess state $(|\beta\rangle)$ which has been phase-encoded by an electro-optic phase modulator. The subtraction stage (enclosed within a dot-dash green line) purifies the quantum states and then send the amplified output to the tomography stage (enclosed within a solid purple line) for fidelity and correct state fraction estimations. 


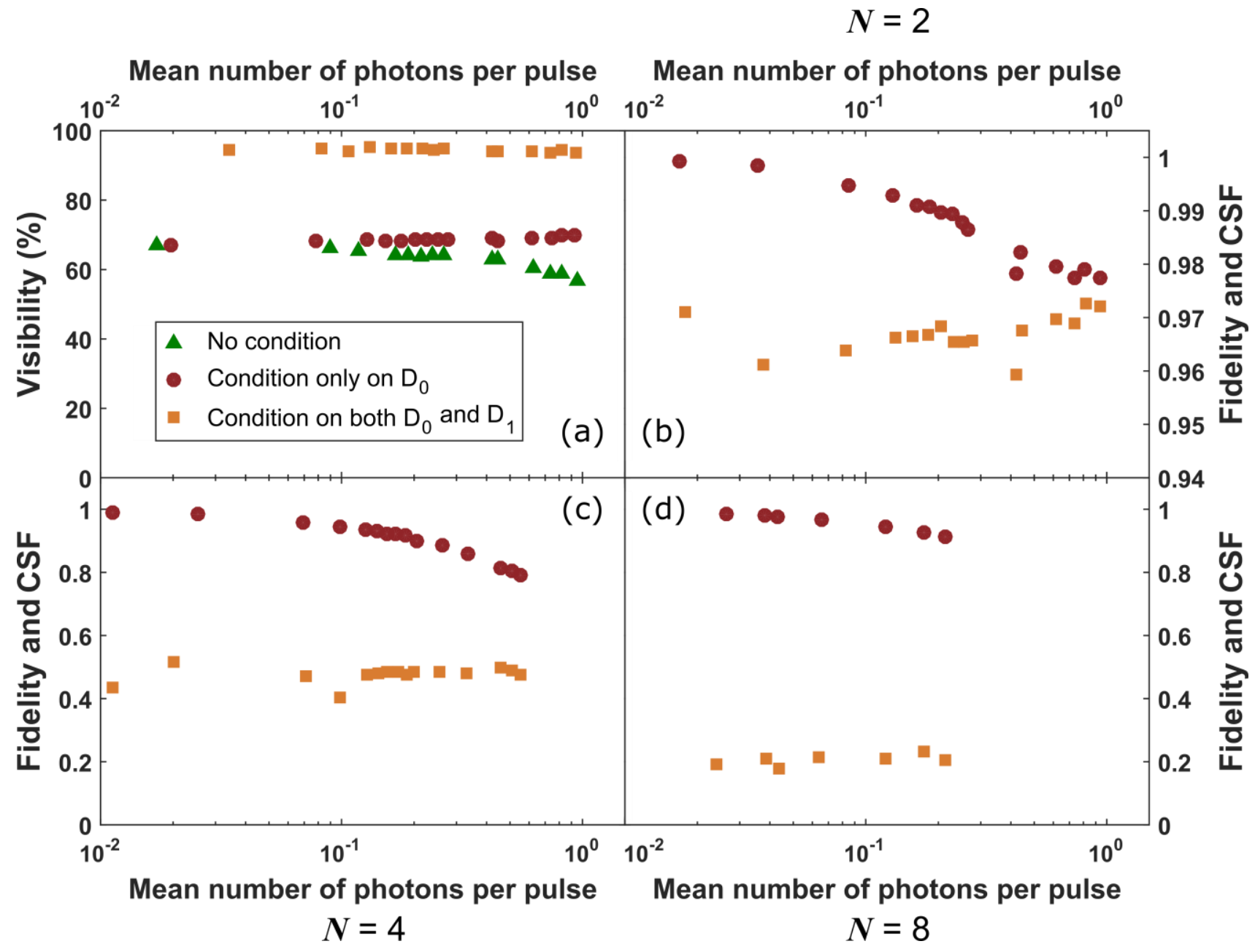

Figure 2. Experimental results of SCAMP. (a) shows the computed visibilities of the tomography stage for $N=2$ when applying three different post-selection criteria: no conditioning (green triangles), conditioning only on detector $\mathrm{D}_{0}$ output (brown dots) and conditioning on both detectors $\mathrm{D}_{0}$ and $\mathrm{D}_{1}$ outputs (orange squares). (b)-(d) show computed fidelity (brown dots) and correct state fraction (CSF) (orange squares) for the cases $N=2,4$ and 8.

Fig. 2 (a) reports the visibility analysis of the outer interferometer, i.e. tomography stage, for $N=2$ as a function of the mean number of photons per pulse, of the coherent states. The plot shows three separate conditioning scenarios on the amplifier's output states: no conditioning (green triangles), conditioning constrained only on detector the output of $\mathrm{D}_{0}$ (brown dots), conditioning constrained by the outputs of both detectors $\mathrm{D}_{0}$ and $\mathrm{D}_{1}$ (orange squares). As expected, incorporating more conditioning constraints on the system increases the fidelity of the output states allowing increasing the visibility measured at the tomography stage. Introducing the state subtraction stage limits the number of wrong states reaching the outer tomography interferometer and improves the overall fidelity of the output state compared to a perfectly amplified version of the input state.

Figs. 2 (b)-(d) show the correct state fraction (CSF) (squares) and fidelity (circles) for $N=2,4$ and 8 . Nominal CSF values for $N=2,4$ and 8 when conditioning criteria are not implemented in the analysis would be $50 \%, 25 \%$ and $12.5 \%$ respectively (that is, equal to the probability of randomly selecting the correct state with a completely uninformed choice), however, the amplification has increased these values to $\approx 97 \%, \approx 60 \%$ and $\approx 30 \%$. Therefore, the SCAMP is more successful than an uninformed random guess. The amplifier not only increases the CSF values but also increases the output fidelity by reducing the probability of amplification of states whose amplitudes are different from the target state. The slight downwards trend of the fidelity with increasing number of photons is attributed to the nature of measurement of an optical pulse by a Si-SPAD. These Geiger-mode devices output a fixed electrical signal irrespective of the incident optical intensity, i.e. number of photons per pulse, therefore, at larger mean number of photons per pulse 
the measured count rates approach an asymptote equal to the system's clock frequency, i.e. $1 \mathrm{MHz}$ restricting the estimation of the fidelity ${ }^{[2]}$. The nominal gain of the system, defined as the ratio of the BS2 transmittivity to the BS1 reflectivity yields a value of 1.8 based on the component's parameters while the maximum measured success probability is $2.6 \%$ at a mean number of photons per pulse of 0.94 .

\subsubsection{High gain SCAMP and a secondary state subtraction stage}

Fig. 3 shows the revised SCAMP optical setup where the 50:50 BS at the state comparison stage has been replaced by a highly transmissive 90:10 BS. This was introduced to increase the overall gain of the SCAMP. An additional state subtraction stage has been introduced for a secondary set of measurements. As in the case of the primary subtraction stage, this secondary subtraction stage comprised a highly transmissive 90:10 BS and a Si-SPAD operated in Geigermode (here denoted by $\mathrm{D}_{2}$ ). The overall operation remained similar to the previous SCAMP configuration and the secondary state subtraction stage provided an additional post-selection constrain on the analysis, i.e. a successful amplification event was now defined when detector $\mathrm{D}_{0}$ at the state comparison stage did not fire and both detectors $\mathrm{D}_{1}$ and $\mathrm{D}_{2}$, at the first and second state subtraction stage respectively, did fire.

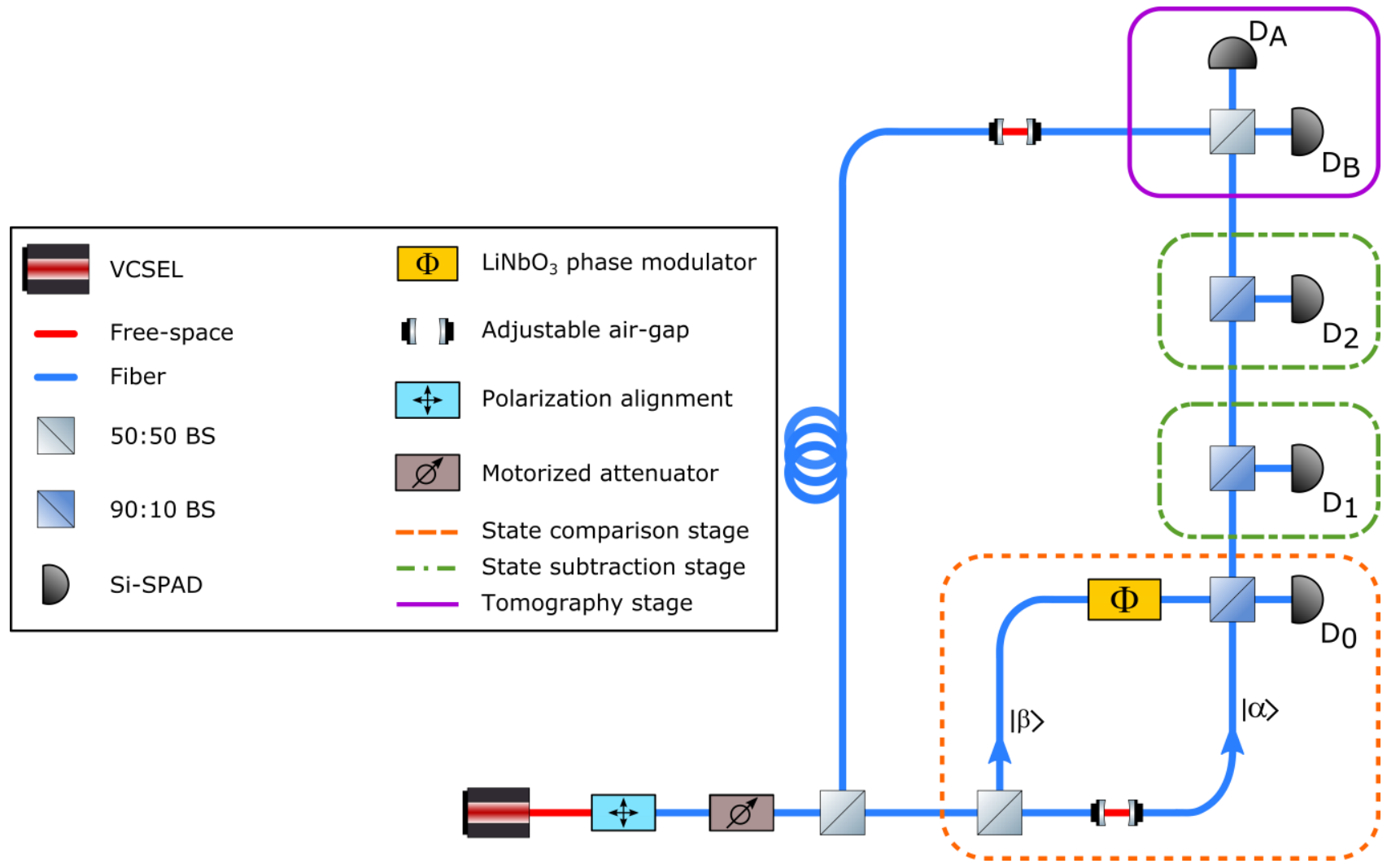

Figure 3. Experimental optical setup of SCAMP with high-transmissive BS and additional subtraction stage. The operating parameters remain the same as for the previous experiment. The additional subtraction stage provides a third post-selection constraint to the analysis refining the system acquisition criteria. In this configuration $(|\alpha\rangle)$ defines the input state and $(|\beta\rangle)$ the guess state which is phase encoded by the lithium-niobate modulator. 


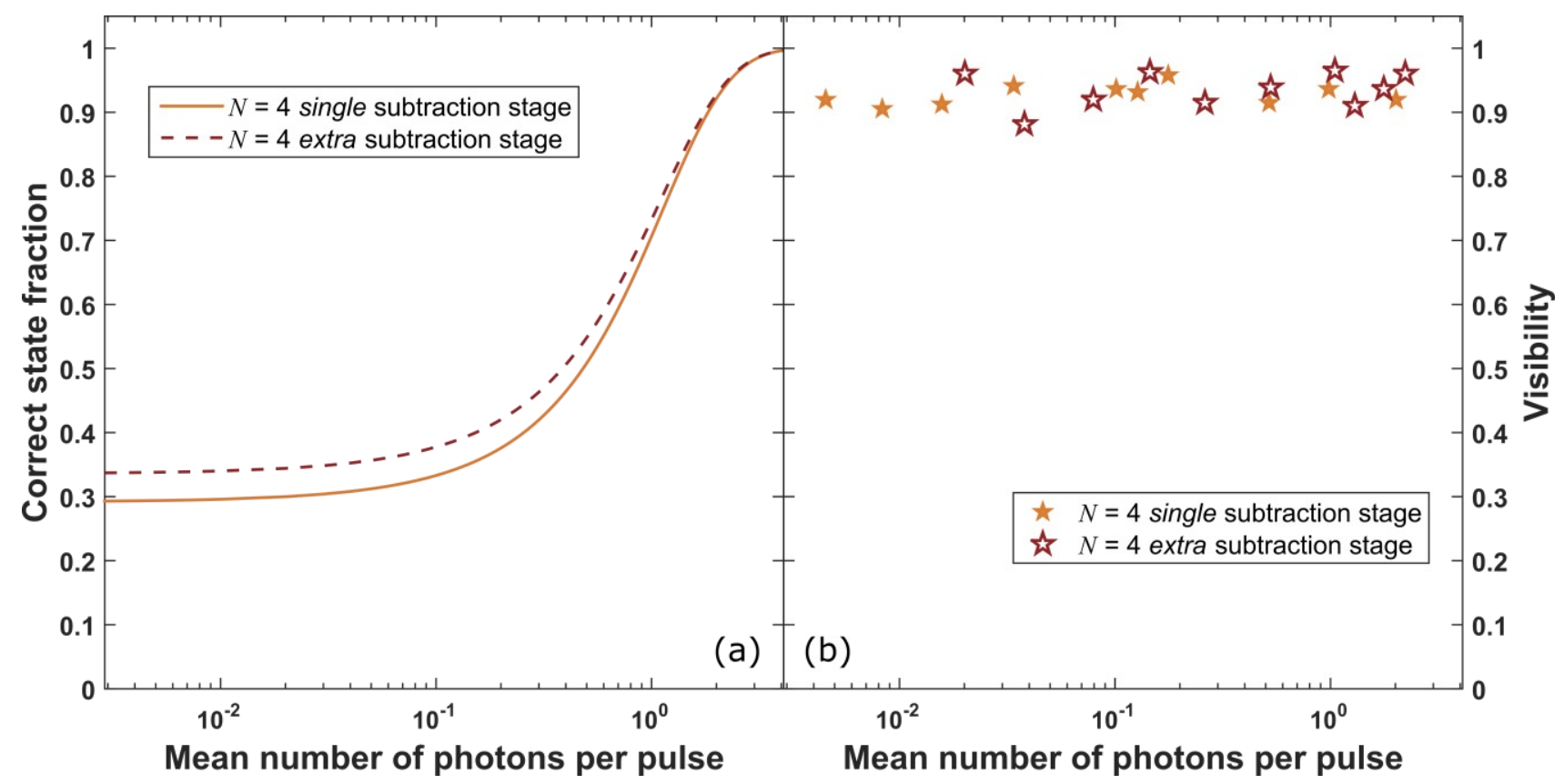

Figure 4. Theoretical and experimental results of the SCAMP with a higher gain, and an additional subtraction stage. (a) shows the theoretical correct state fraction with (dashed brown line) and without (solid orange line) the additional state subtraction stage for $N=4$. (b) shows the visibility of the tomography stage for the same $N=4$ case. A visibility of more than $90 \%$ suggests the fidelity of the amplified states will be high.

Figs. 4 (a)-(b) report the theoretical and experimental results of both configurations, i.e. the optical setup with a highly transmissive 90:10 BS at the state comparison stage and the other setup with an additional state subtraction stage for $N=4$. Fig. 4 (a) clearly shows an improvement over the correct state fraction (dashed brown line) when a secondary state subtraction stage is added to the system. This improvement comes at the cost of success probability, due to the additional post-selection condition. The visibility of the outer interferometer (tomography stage) reaches values as high as $97 \%$ demonstrating high contrast between a correctly chosen guess state and a wrongly chosen guess state (see Fig. 4 (b)) in both configurations. The nominal gain for the 90:10 BS was given to be 8.35 , and 6.5 with the additional subtraction stage. These were derived by including unbalanced beam-splitting ratios of the optical components to the analysis.

\subsection{SCAMP with added channel noise}

Fig. 5 shows the SCAMP configuration used to evaluate the amplifier's performance and robustness against background noise. This background noise might be present in real applications when the device is deployed across fiber telecommunication networks, and could be due to ambient light, nonlinear Raman scattering in optical fibers or simply increased detector dark counts. In this experiment, the extra noise was introduced using a light emitting diode (LED) with a central wavelength of $869.5 \mathrm{~nm}$ and spectral linewidth of $31.5 \mathrm{~nm}$ evanescently coupled into an exposed fiber splice situated where the input state is fed to the state comparison stage (Point A). The LED's optical emission was operated in continuous wave $(\mathrm{CW})$ mode in order to send a constant photon flux across the interferometer providing an easy estimation of the background noise when post-selecting on the Si-SPADs detection events. By changing the LED's power output three different noise levels were tested, i.e. low, medium and high noise corresponding to raw count rates of $0.16,0.4$ and 0.8 mega-counts per second respectively at the detector $\mathrm{D}_{0}$. 


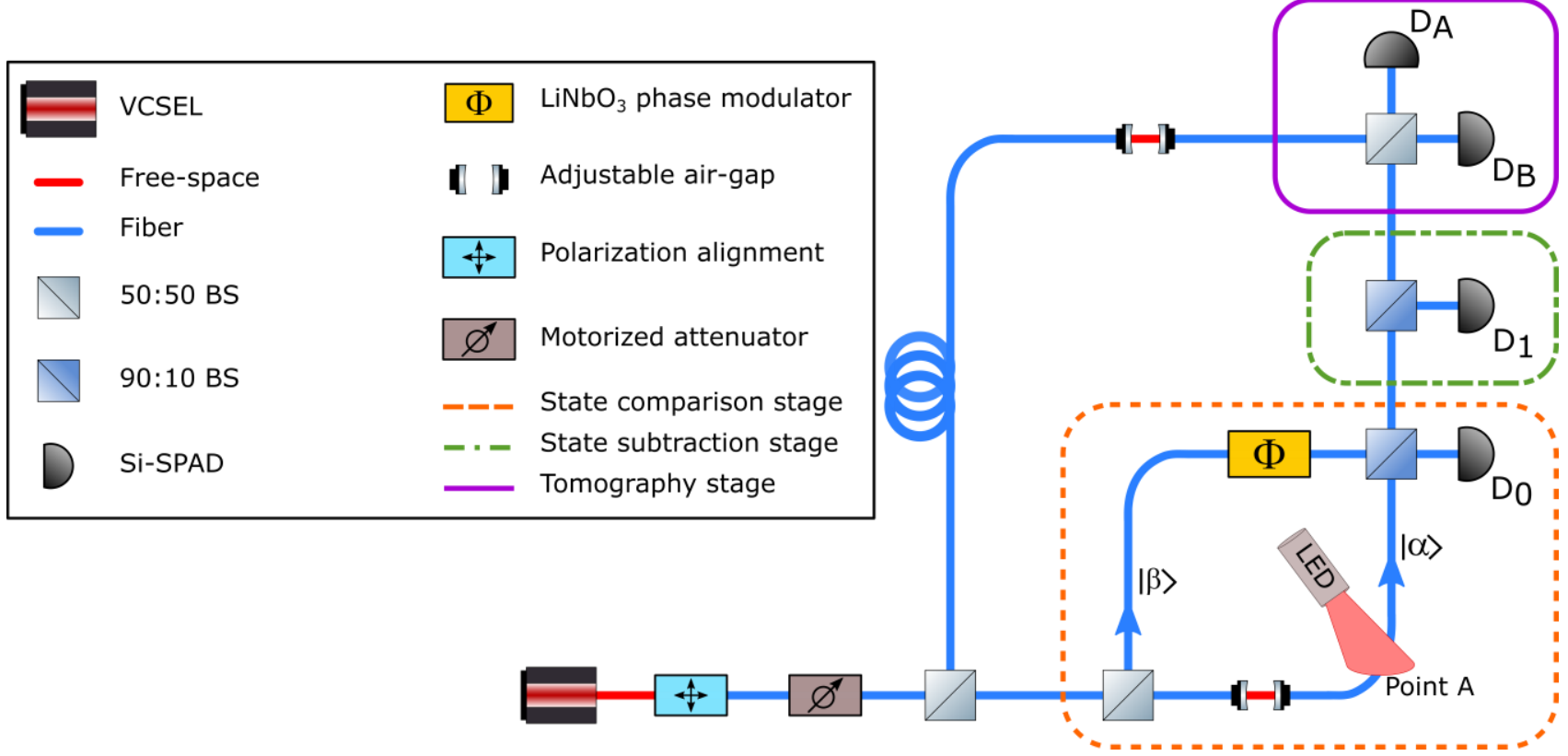

Figure 5. Experimental optical setup of SCAMP with added channel noise. An LED source, operating in CW mode, is coupled into an exposed fiber splice (Point A) where the input signal is fed to the state comparison stage. The broadband noise can be varied by changing the LED output power. The state $(|\alpha\rangle)$ defines the input while $(|\beta\rangle)$ the guess state.

Figs. 6 (a)-(b) report the experimental results showing a comparison of the amplifier's parameters, i.e. state fraction and visibility, with and without added channel noise. To improve the readability of the graphs Fig. 6 (a) shows only data points relative to the mid-noise levels for $N=4$. Similarly, Fig. 6 (b) only shows data points for $N=4$, covering the entire noise level range, as the cases $N=2$ and 8 display similar trends.

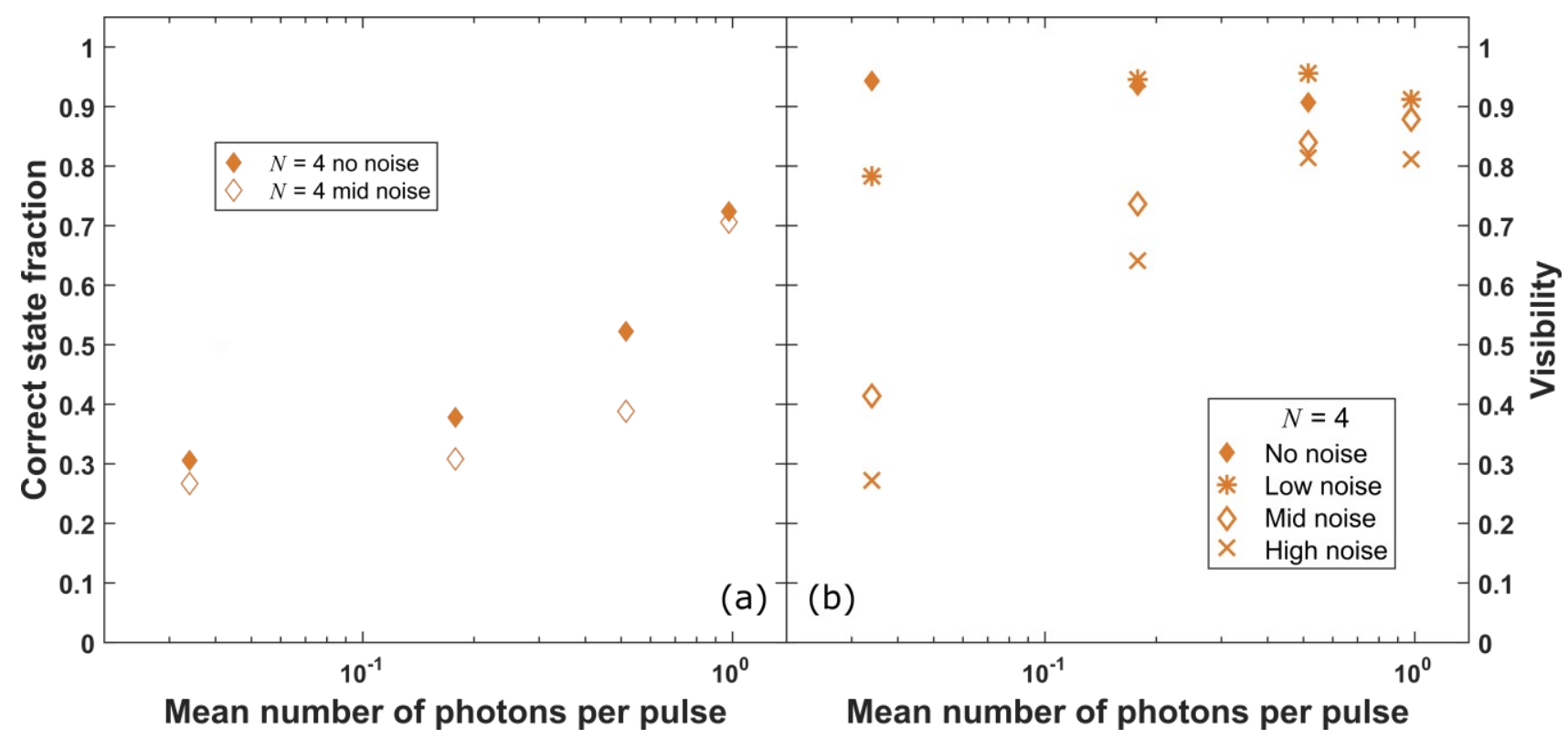

Figure 6. Experimental results of SCAMP with added channel noise. (a) shows the computed correct state fraction with (hollow data points) and without (filled data points) the external broadband noise introduced by the LED source for $N=4$. (b) shows the visibility of the tomography stage for the same $N=4$ case and different noise levels. 
The correct state fraction shows a decrease at lower numbers of photons because, although more photons reach the subtraction stage due to the added external broadband noise, incorrect states have a higher probability of being detected by $\mathrm{D}_{1}$ and erroneously triggering the system's post-selection criteria. As the mean number of photons per pulse increases, the results converge to the no-noise case due to an increased probability of a wrong state triggering detector $\mathrm{D}_{0}$ at the state comparison stage, thereby lowering the number of spurious events that meet the system's post-selection criteria. To provide a performance estimation, Fig. 6 (b) shows the visibility computed for the tomography stage, highlighting the contribution noise makes to the system. As expected, the addition of noise negatively affects the system at low mean photon numbers, i.e. less than 0.5. At higher photon number values the visibility is partially recovered because of the increased signal to noise ratio (SNR), however quantum experiments typically operate at low mean photon numbers ${ }^{[32]}$, e.g. less than 0.5. A visibility estimation for the amplifier can, in theory, provide valuable information on possible external accesses to the device by a malicious party and may be useful in identifying security related attacks such as Trojan horse attack or detector blinding attack ${ }^{[33],[34]}$.

\section{CONCLUSION AND FUTURE WORK}

This paper has demonstrated an easy way to implement quantum amplifier based on a state comparison technique for a discrete set of phase encoded optical quantum states. The amplifier here greatly surpasses alternative probabilistic amplifiers in terms of output fidelity, success probability and gain ${ }^{[18],[20],[35],[36]}$ using coherent states, linear optical components and commercially available single-photon detectors. Three different SCAMP configurations were described: namely "basic" SCAMP ${ }^{[27]}$, SCAMP with an extra subtraction state, and a SCAMP operating in the presence of additional external noise. The first configuration of SCAMP provided benchmark values for state comparison amplification based on the photon addition and subtraction procedure. The system showed a success probability as high as $2.6 \%$ and approximately a twofold increase over the measured correct state fractions compared to the nominal values from a uniformed random guess strategy. A secondary SCAMP configuration with an added subtraction stage showed enhanced gain and correct state fraction. The same configuration was also tested for robustness against coupled external ambient light, crosstalk from co-propagating signal channels or excessively noisy detectors showing the ability of the device to perform in non-ideal environmental conditions.

All of the SCAMP configurations discussed in this paper implement a series of post-condition criteria to discriminate successful amplifications event from spurious ones by considering cross correlation of detection events. However, events where the amplifier has made a wrong guess when comparing the quantum states at the state comparison stage are discarded and this affects both the performance and efficiency of the amplifier. One possible way to address the problem is to actively change the guess state by implementing a secondary state comparison stage using a feedforward mechanism based on the response of detector $\mathrm{D}_{0}$. It is hypothesized that the feedforward mechanism would increase the success probability and output fidelity.

The modular nature of the SCAMP makes it easily reconfigurable and therefore easy to adapt for different applications. However, it is currently not a compact system due to the many beam splitters and optical components which compose the different stages and therefore the output is easily influenced by thermal and mechanical stress. We believe that many of these problems can potentially be resolved by miniaturizing the device onto a photonic integrated circuit.

\section{REFERENCES}

[1] Bennett, C. H. and Brassard, G., "Quantum cryptography: Public key distribution and coin tossing", IEEE International Conference on Computers, Systems and Signal Processing Proceedings 175, 8 (1984)

[2] Gisin, N., Ribordy, G., Tittle, W. and Zbinden, H., "Quantum cryptography", Rev. Mod. Phys. 74, 145 (2002)

[3] Korzh, B., Lim, C. C. W., Houlmann, R., Gisin, N., Li, M. J., Nolan, D., Sanguinetti, B., Thew, R. and Zbinden, H., "Provably secure and practical quantum key distribution over $307 \mathrm{~km}$ of optical fibre", Nat. Photonics 9, 163-168 (2015)

[4] Gleim, A. V., Egorov, V. I., Nazarov, Yu. V., Smirnov, S. V., Chistyakov, V. V., Bannik, O. I., Anisimov, A. A., Kynev, S. M., Ivanova, A. E., Collins, R. J., Kozlov, S. A. and Buller, G. S., "Secure polarizationindependent subcarrier quantum key distribution in optical fiber channel using BB84 protocol with a strong reference", Opt. Express 24, 2619-2633 (2016) 
[5] Clarke, P. J., Collins, R. J., Dunjko, V., Andersson, E., Jeffers, J. and Buller, G. S., "Experimental demonstration of quantum digital signatures using phase-encoded coherent states of light", Nat. Commun. 3, 1174 (2012)

[6] Collins, R. J., Donaldson, R. J., Dunjko, V., Wallden, P., Clarke, P. J., Andersson, E., Jeffers, J. and Buller, G. S., "Realization of Quantum Digital Signatures without the Requirement of Quantum Memory", Phys. Rev. Lett. 113, 040502 (2014)

[7] Donaldson, R. J., Collins, R. J., Kleczkowska, K., Amiri, R., Wallden, P., Dunjko, V., Jeffers, J., Andersson, E. and Buller, G. S., "Experimental demonstration of kilometer-range quantum digital signatures", Phys. Rev. A 93, $012329(2016)$

[8] Collins, R. J., Amiri, R., Fujiwara, M., Honjo, T., Shimizu, K., Tamaki, K., Takeoka, M., Andersson, E., Buller, G. S. and Sasaki, M., "Experimental transmission of quantum digital signatures over $90 \mathrm{~km}$ of installed optical fiber using a differential phase shift quantum key distribution system", Opt. Lett. 41, 4883-4886 (2016)

[9] Collins, R. J., Amiri, R., Fujiwara, M., Honjo, T., Shimizu, K., Tamaki, K., Takeoka, M., Sasaki, M., Andersson, E. and Buller, G. S., "Experimental demonstration of quantum digital signatures over $43 \mathrm{~dB}$ channel loss using differential phase shift quantum key distribution", Sci. Rep. 7, 3235 (2017)

[10]Liu, Y., Cao, Y., Curty, M., Liao, S.-H., Wang, J., Cui, K., Li, Y.-H., Lin, Z.-H., Sun, Q.-C., Li, D.-D., Zhang, H.-F., Zhao, Y., Chen, T.-Y., Peng, C.-Z., Zhang, Q., Cabello, A. and Pan, J.-W., "Experimental Unconditionally Secure Bit Commitment", Phys. Rev. Lett. 112, 010504 (2014)

[11] Ter-Mikirtychev, V., [Main Optical Components for Fiber Laser/Amplifier Design], Springer, Cham, 133-160 (2014)

[12] Caves, C. M., "Quantum limits on noise in linear amplifiers", Phys. Rev. D. 26, 1817 (1982)

[13] Lucamarini, M., Patel, K. A., Dynes, J. F., Fröhlich, B., Sharpe, A. W., Dixon, A. R., Yuan, Z. L., Penty, R. V. and Shields, A. J., "Efficient decoy-state quantum key distribution with quantified security", Opt. Express 21, 24550-24565 (2013)

[14] Shibata, H., Honjo, T. and Shimizu, K., "Quantum key distribution over a 72 dB channel loss using ultralow dark count superconducting single-photon detectors", Opt. Lett. 39, 5078-5081 (2014)

[15] Wootters, W. K. and Zurek, W. H., "A single quantum cannot be cloned", Nat. 299, 802-803 (1982)

[16] Ralph, T. C. and Lund, A. P., "Nondeterministic Noiseless Linear Amplification of Quantum Systems", AIP Conference Proceedings 1110, 155 (2009)

[17] Fiurášek, J., "Engineering quantum operations on traveling light beams by multiple photon addition and subtraction", Phys. Rev. A 80, 053822 (2009)

[18] Müller, C. R., Wittmann, C., Marek, P., Filip, R., Marquardt, C., Leuchs, G. and Andersen, U. L., "Probabilistic cloning of coherent states without a phase reference", Phys. Rev. A 86, 010305 (2012)

[19] Marek, P. and Filip, R., "Coherent-state phase concentration by quantum probabilistic amplification", Phys. Rev. A 81, 022302 (2010)

[20]Zavatta, A., Fiurášek, J. and Bellini, M., "A high-fidelity noiseless amplifier for quantum light states", Nat. Photonics 5, 52-60 (2011)

[21]Pegg, D. T., Phillips, L. S. and Barnett, S. M., "Optical State Truncation by Projection Synthesis", Phys. Rev. Lett. 81, 1604 (1998)

[22] Gisin, N., Pironio, S. and Sangouard, N., "Proposal for Implementing Device-Independent Quantum Key Distribution Based on a Heralded Qubit Amplifier", Phys. Rev. Lett. 105, 070501 (2010)

[23] Fasel, S., Gisin, N., Ribordy, G., Scarani, V. and Zbinden, H., "Quantum Cloning with an Optical Fiber Amplifier", Phys. Rev. Lett. 89, 107901 (2002)

[24] Wang, S., Chen, W., Guo, J.-F., Yin, Z.-Q., Li, H.-W., Zhou, Z., Guo, G.-C. and Han, Z.-F., "2 GHz clock quantum key distribution over $260 \mathrm{~km}$ of standard telecom fiber", Opt. Lett. 37, 1008-1010 (2012)

[25] Eleftheriadou, E., Barnett, S. M. and Jeffers, J., "Quantum Optical State Comparison Amplifier", Phys. Rev. Lett. 111, 213601 (2013)

[26] Chefles, A., Andersson, E. and Jex, I., "Unambiguous comparison of the states of multiple quantum systems", J. Phys. A: Math. Gen. 37, 7315 (2004)

[27] Donaldson, R. J., Collins, R. J., Eleftheriadou, E., Barnett, S. M., Jeffers, J. and Buller, G. S., "Experimental Implementation of a Quantum Optical State Comparison Amplifier", Phys. Rev. Lett. 114, 120505 (2015)

[28] Gordon, K. J., Fernandez, V., Townsend, P. D. and Buller, G. S., "A short wavelength GigaHertz clocked fiberoptic quantum key distribution system", IEEE J. Quantum Electron. 40, 900-908 (2004) 
[29]Buller, G. S. and Collins, R. J., "Single-photon generation and detection", Meas. Sci. Technol. 21, 012002 (2010)

[30] Collins, R. J., Hadfield, R. H. and Buller, G. S., "Commentary: New developments in single photon detection in the short wavelength infrared regime", J. Nanophotonics 4, 040301 (2010)

[31] Clarke, P. J., Collins, R. J., Hiskett, P. A., Martínez, M. J. G., Krichel, N. J., McCarthy, A., Tanner, M. G., O’Connor, J. A., Natarajan, C. M., Miki, S., Sasaki, M., Wang, Z., Fujiwara, M., Rech, I., Ghioni, M., Gulinatti, A., Hadfield, R. H., Townsend P. D. and Buller, G. S., "Analysis of detector performance in a gigahertz clock rate quantum key distribution system", New J. Phys. 13, 075008 (2011)

[32] Huttner, B., Imoto, N., Gisin, N. and Mor, T., "Quantum cryptography with coherent states", Phys. Rev. A 51, 1863 (1995)

[33] Lydersen, L., Wiechers, C., Wittmann, C., Elser, D., Skaar, J. and Makarov, V., "Thermal blinding of gated detectors in quantum cryptography", Opt. Express 18, 27938-27954 (2010)

[34] Gisin, N., Fasel, S., Kraus, B., Zbinden, H. and Ribordy, G., "Trojan-horse attacks on quantum-key-distribution systems", Phys. Rev. A 73, 022320 (2006)

[35] Xiang, G. Y., Ralph, T. C., Lund, A. P., Walk, N. and Pryde, G. J., "Heralded noiseless linear amplification and distillation of entanglement", Nat. Photonics 4, 316-319 (2010)

[36] Ferreyrol, F., Barbieri, M., Blandino, R., Fossier, S., Tualle-Brouri, R. and Grangier, P., "Implementation of a Nondeterministic Optical Noiseless Amplifier", Phys. Rev. Lett. 104, 123603 (2010) 